\title{
Comprehensive analysis of long non-coding RNA s and mRNAs in skeletal muscle of diabetic Goto-Kakizaki rats during the early stage of type 2 diabetes
}

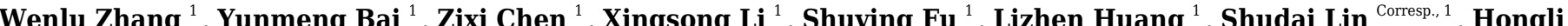
Du ${ }^{\text {Corresp. } 1}$

${ }^{1}$ School of Biology and Biological Engineering, South China University of Technology, Guangzhou, China

Corresponding Authors: Shudai Lin, Hongli Du

Email address: linsd@scut.edu.cn, hldu@scut.edu.cn

Skeletal muscle long non-coding RNAs (IncRNAs) were reported to be involved in the development of type 2 diabetes (T2D). However, little is known about the mechanism of skeletal muscle IncRNAs on hyperglycemia of diabetic Goto-Kakizaki (GK) rats at the age of 3 and 4 weeks. To elucidate this, we used RNA-sequencing to profile the skeletal muscle transcriptomes including IncRNAs and mRNAs, in diabetic GK and control Wistar rats at the age of 3 and 4 weeks. Totally, there were 438 differentially expressed mRNAs (DEGs) and 401 differentially expressed IncRNAs (DELs) in skeletal muscle of 3-week-old GK rats compared with age-matched Wistar rats, and 1000 DEGs and 726 DELs between GK rats and Wistar rats at 4 weeks of age. The protein-protein interaction analysis of overlapping DEGs between 3 and 4 weeks, the correlation analysis of DELs and DEGs, as well as the prediction of target DEGs of DELs showed that these DEGs (Pdk4, Stc2, II15, Fbxw7 and Ucp3) might play key roles in hyperglycemia, glucose intolerance, and increased fatty acid oxidation. Considering the corresponding co-expressed DELs with high correlation coefficients or targeted DELs of these DEGs, our study indicated that these dysregulated IncRNA-mRNA pairs (NONRATG017315.2-Pdk4, NONRATG003318.2-Stc2, NONRATG011882.2-II15, NONRATG013497.2-Fbxw7, MSTRG.1662-Ucp3) might be related to above biological processes in GK rats at the age of 3 and 4 weeks. Our study could provide more comprehensive knowledge of mRNAs and IncRNAs in skeletal muscle of GK rats at 3 and 4 weeks of age. And our study may provide deeper understanding of the underlying mechanism in T2D of GK rats at the age of 3 and 4 weeks. 


\section{Comprehensive analysis of long non-coding RNAs and mRNAs in}

2 skeletal muscle of diabetic Goto-Kakizaki rats during the early stage

3 of type 2 diabetes

4 Wenlu Zhang, Yunmeng Bai, Zixi Chen, Xingsong Li, Shuying Fu, Lizhen Huang, Shudai

5 Lin* and Hongli Du*

6

7 School of Biology and Biological Engineering, South China University of Technology,

8 Guangzhou, Guangdong, China.

9

$10 *$ Corresponding authors:

11 Hongli Du, hldu@scut.edu.cn

12 Shudai Lin, linsd@scut.edu.cn

13 School of Biology and Biological Engineering, South China University of Technology, 382

14 Zhonghuan Road East, Panyu District, Guangzhou Higher Education Mega Centre, Guangzhou 15 510006, Guangdong, China 


\section{Abstract}

18 Skeletal muscle long non-coding RNAs (lncRNAs) were reported to be involved in the development of type 2 diabetes (T2D). However, little is known about the mechanism of skeletal muscle lncRNAs on hyperglycemia of diabetic Goto-Kakizaki (GK) rats at the age of 3 and 4 weeks. To elucidate this, we used RNA-sequencing to profile the skeletal muscle transcriptomes including lncRNAs and mRNAs, in diabetic GK and control Wistar rats at the age of 3 and 4 weeks. Totally, there were 438 differentially expressed mRNAs (DEGs) and 401 differentially expressed lncRNAs (DELs) in skeletal muscle of 3-week-old GK rats compared with agematched Wistar rats, and 1000 DEGs and 726 DELs between GK rats and Wistar rats at 4 weeks of age. The protein-protein interaction analysis of overlapping DEGs between 3 and 4 weeks, the correlation analysis of DELs and DEGs, as well as the prediction of target DEGs of DELs showed that these DEGs (Pdk4, Stc2, Il15, Fbxw7 and Ucp3) might play key roles in hyperglycemia, glucose intolerance, and increased fatty acid oxidation. Considering the corresponding co-expressed DELs with high correlation coefficients or targeted DELs of these DEGs, our study indicated that these dysregulated lncRNA-mRNA pairs (NONRATG017315.2Pdk4, NONRATG003318.2-Stc2, NONRATG011882.2-Il15, NONRATG013497.2-Fbxw7, MSTRG.1662-Ucp3) might be related to above biological processes in GK rats at the age of 3 and 4 weeks. Our study could provide more comprehensive knowledge of mRNAs and lncRNAs in skeletal muscle of GK rats at 3 and 4 weeks of age. And our study may provide deeper understanding of the underlying mechanism in T2D of GK rats at the age of 3 and 4 weeks.

\section{7}

\section{Introduction}

It has been demonstrated that approximately $75 \%$ human genome is transcribed, and nearly 97\% genomic DNA cannot be translated into proteins (Djebali et al. 2012). These RNAs without protein-coding ability are known as non-coding RNAs (ncRNAs). Among them, long ncRNAs (lncRNAs) are more than 200 nucleotides in length (Esteller 2011; Guttman \& Rinn 2012), exhibiting tissue-specific (Cabili et al. 2011; Mercer et al. 2008; Tsoi et al. 2015) and low 
43 expression levels (Derrien et al. 2012). They could promote (Guil \& Esteller 2012; Luo \& Chen 44 2016) or suppress (Espinoza et al. 2004; Peterlin et al. 2012; Rinn et al. 2007) the expression 45 level of their target genes. Furthermore, it has been revealed that the expression of lncRNAs was 46 dysregulated in many diseases, such as type 2 diabetes (T2D) (Akerman et al. 2017; Liu et al. 47 2014; Reddy et al. 2014).

48 As it was uncovered, IncRNAs were closely correlated to T2D. Upregulated expression of 49 lncRNA Meg3 could contribute to insulin resistance in $o b / o b$ mice liver (Zhu et al. 2016). The $50 d b / d b$ mice islets showed significantly decreased expression of lncRNA Meg3, and the islet51 specific knockdown of lncRNA Meg3 resulted in less insulin synthesis and secretion but larger 52 scale of $\beta$ cell apoptosis, consequently lead to impaired glucose tolerance (You et al. 2016). The 53 islet-specific lncRNA Tug1 knockdown mice exhibited an increased apoptosis ratio and a lower 54 insulin secretion in the $\beta$ cells (Yin et al. 2015; You et al. 2016). Besides, the reduced expression 55 of lncRNA H19 could impair insulin sensitivity and decrease glucose uptake in muscle cells 56 (Gao et al. 2014). Moreover, significantly decreased expression of H19 was observed in muscle 57 of T2D patients (Gao et al. 2014), suggesting the importance of skeletal muscle lncRNAs to the 58 development of T2D. As one of the target tissues of insulin, skeletal muscle is burdened with $5970 \%-80 \%$ postprandial glucose disposal responsibility (Baron et al. 1988; DeFronzo et al. 1981). 60 Therefore, lncRNAs in skeletal muscle might play critical roles in regulating whole-body 61 glucose homeostasis and T2D development.

62 As a non-obese model for T2D, Goto-Kakizaki (GK) rats are produced by selective

63 breeding from Wistar rats with impaired glucose tolerance (Goto et al. 1976; Kitahara et al. 64 1978). GK rats show postprandial glucose intolerance and insulin resistance in skeletal muscle 65 and adipose tissue (Bisbis et al. 1993; Portha et al. 2012), and exhibit hyperglycemia during age 66 of 3-4 weeks (Ando et al. 2018). Though GK rats has been found to exhibit defects in skeletal 67 muscle and their related mRNA expression level has been investigated (Dadke et al. 2000; 68 Steiler et al. 2003), the regulation mechanism of skeletal muscle lncRNA to postprandial 69 hyperglycemia in GK rat at the age of 3 and 4 weeks is still indistinct. 
70

71

72

73

74

75

76

77

78

79

80

81

82

83

84

85

86

87

88

89

90

91

92

93

94

To explore the role of skeletal muscle lncRNAs in hyperglycemia development, we compared the skeletal muscle transcriptomes between T2D GK rats and control Wistar rats, to find out the differentially expressed mRNAs (DEGs) and differentially expressed lncRNAs (DELs). Subsequently, we conducted protein-protein interaction analysis, screened the coexpressed lncRNA-mRNA pairs with high correlation coefficients, and predicted the target mRNAs of DELs and the target microRNAs (miRNAs) of key DEGs and DELs. Our results suggested that the dysregulated lncRNAs might be implicated in hyperglycemia, glucose intolerance, as well as dysregulated glucose and fatty acid oxidation in skeletal muscle of GK rats at the age of 3 and 4 weeks. These findings might help us understand more about the regulation mechanism of skeletal muscle lncRNAs in T2D development.

\section{Materials and methods}

\section{Ethical approval}

22 The study was approved by the institutional review board of the Guangdong Key Laboratory of Laboratory Animals. All protocols were carried out in accordance with the guidelines of the Institutional Animal Care and Use Committee (IACUC) [Ethics certificate No.: IACUC2014029].

\section{Animal breeding and tissues samples collection}

Four groups of rats (diabetic male GK rats and control male Wistar rats at 3 weeks of age, diabetic male GK rats and diabetic male GK rats at 4 weeks of age, $n=10$ each group), totally 40 subjects were used in this study. Rats were raised in a room with 12 hours dark: 12 hours light cycle, 20 to $25^{\circ} \mathrm{C}$ temperature and $60 \pm 5 \%$ humidity, at the SLAC Laboratory Animal Co., Ltd. (Shanghai, China) (Almon et al. 2012; Nie et al. 2017; Nie et al. 2011; Xue et al. 2011). All animals were free access to food and water. Body weight of each rat was measured weekly by weighing. Food disappearance was measured by weighing the difference in the weight of feed added and the feed remaining. The behavior of rats including feeding, drinking, sleeping and 
95 digging were observed. Blood samples were collected from the orbital plexus veins behind the 96 eyeball using EDTA (4 mM final concentration) as an anticoagulant. Plasma was obtained from

97 blood after centrifugation $\left(2000 \times \mathrm{g}, 4^{\circ} \mathrm{C}, 15 \mathrm{~min}\right)$, divided into aliquots, and then stored at $-80^{\circ} \mathrm{C}$.

98 All rats were administered anesthesia with pentobarbital sodium (intraperitoneal, $50 \mathrm{mg} / \mathrm{kg}$ body 99 weight), then were killed by cervical dislocation. Samples of gastrocnemius muscle of each rats 100 were harvested, followed by rapidly frozen in liquid nitrogen, and stored at $-80^{\circ} \mathrm{C}$ for future 101 studies (Nie et al. 2017; Nie et al. 2011). Six gastrocnemius muscle samples from six rats each 102 group were selected randomly for RNA-sequencing in the present study.

103

104 105

\section{Measurement of plasma glucose and insulin concentration}

The automatic Dry Biochemical Analyzer FUJIFILM DRI-CHEM 7000i with GLU-PIII slides (Fujifilm, Saitama, Japan) was used to measure random plasma glucose concentration. And Thermo scientific Rat Insulin ELISA Kit (Cat\#ERINS, Invitrogen, Waltham, MA, USA) was used to measured plasma insulin concentration. Assays were conducted according to the manufacturer's instructions.

\section{RNA extraction and sequencing}

Total RNA for RNA-sequencing was extracted from red part of each gastrocnemius muscle using TRIzol Reagent (Cat\#15596-018, Life Technologies, Carlsbad, CA, USA) following the manufacturer's instructions. RNA integrity and concentration were measured by the Bioanalyzer 2100 system (Agilent Technologies, Santa Clara, CA, USA). Ribosomal RNA was removed using Epicentre Ribo-Zero ${ }^{\mathrm{TM}}$ Gold Kits (Epicentre, Madison, WI, USA) according to the manufacturer's instructions. RNA-sequencing was performed on Illumina HiSeq X Ten system (Illumina) following the HiSeq X Ten User Guide to generate $150 \mathrm{bp}$ paired-end reads. 
117

118

119

120

121

122

123

124

125

126

127

128

129

130

131

132

133

134

135

136

137

138

139

140

141

142

143

Analysis of differentially expressed $m R N A$ s and lncRNAs

After quality control and filtering of low quality reads, we used STAR (Dobin \& Gingeras 2015) version 020201 to align the cleaned reads of each sample to the Rattus norvegicus reference genome (Ensembl Rnor_6.0 version 92) with the parameters of --quantMode GeneCounts --outSAMstrandField intronMotif --outSAMtype BAM SortedByCoordinate -outSAMtype BAM SortedByCoordinate --twopassMode Basic. All the corresponding annotation files of Rattus_norvegicus.Rnor_6.0.92.gtf (ftp://ftp.ensemb1.org/pub/release-

92/gtf/rattus_norvegicus) and NONCODEv5_rat_rn6_lncRNA.gtf were downloaded from the Ensembl database54 and NONCODE version v5.0

(http://www.noncode.org/datadownload/NONCODEv5 rat rn6 lncRNA.gtf.gz), respectively. Cufflinks were used for alignment of novel transcripts. Then the coding-probability of novel transcripts were identified by CPC2 (Kang et al. 2017), CPAT (Wang et al. 2013b) and CNCI (Sun et al. 2013). The novel transcripts with low coding-probability, or without codingprobability should meet the criteria: coding_probability score less than 0.5 in CPC2 and CPAT, and identified as noncoding by CNCI. Those novel transcripts meet criteria above with $\geq 200 \mathrm{bp}$ in length and at least two exons, were defined as novel lncRNAs. Stringtie (Pertea et al. 2015) version 1.3.0 was used to assemble novel lncRNAs, annotated lncRNAs and annotated mRNAs transcripts. The novel lncRNAs were shown in Table S1. Ballgown R package (Frazee et al. 2015) version 2.10.0 was used to estimate the fragments per kilobase of exon per million fragments mapped (FPKM) of lncRNAs and mRNAs. The lncRNAs and mRNAs were filtered with FPKM < 0.5 (Moran et al. 2012). The FPKM from four groups of rats correspond to normal distribution based on the shapiro.test of Shapiro-Wilk test. The normal distribution of FPKM of four groups of rats were shown in Table S2. Next, the FPKM of GK rats at the age of 3 weeks were compared to Wistar rats at the age of 3 weeks, and GK rats at the age of 4 weeks were compared to Wistar rats at the age of 4 weeks. Thus, the differentially expressed lncRNAs (DELs) and differentially expressed mRNAs (DEGs) were obtained by Bayes-regularized t-test with an false discovery rate (FDR) correction using Cyber-T bayesreg (Kayala \& Baldi 2012). 
144 FDR $<0.05$ was regarded as statistically significant. The power of test was calculated by 145 pwr.t.test in R package pwr. The flowchart of data analysis was shown in Fig. 1.

146 Analysis of KEGG pathways and $G O$

147 A Database for Annotation, Visualization and Integrated Discovery (DAVID) version 6.8

148 was used to obtain the Kyoto Encyclopedia of Genes and Genomes (KEGG) pathways and

149 biological process in Gene Ontology (GO). The statistical significance threshold was $P<0.05$.

150

151

152

153

154

155

156

157

158

159

160

161

162

163

164 165

\section{Protein-protein interaction}

An online database resource Search Tool for the Retrieval of Interacting Genes (STRING) (Szklarczyk et al. 2011) version 11.0 was performed to analyze protein-protein interaction of overlapping upregulated mRNAs and downregulated mRNAs between 3 and 4 weeks, respectively. After filtering disconnected nodes, we selected the minimum confidence score above 0.4 of the interaction. The confidence score was a combined score of neighborhood on chromosome, gene fusion, phylogenetic cooccurrence, homology, co-expression, experimentally determined interaction, database annotated, and automated text-mining. Those connected nodes with confidence score were downloaded for constructing the networks of protein-protein interaction.

\section{The correlation analysis of lncRNAs and $m R N A s$}

Python version 3.6.4 was conducted to calculate the Pearson correlation coefficients of lncRNAs and mRNAs. Those selected co-expressed lncRNA-mRNA pairs met the following criteria: correlation coefficient value $>0.9$, and the absolute fold change of these DEGs and DELs $\geq 1.5$. Then, pwr.r.test in R package pwr was carried out to calculate the power of the correlation. 
166

167 Since over 65\% of lncRNAs were located within $10 \mathrm{~kb}$ of genes (Jia et al. 2010), we 168 utilized the University of California Santa Cruz (UCSC) genome browser to identify potential 169 cis-target genes located within $10 \mathrm{~kb}$ of lncRNAs (Liang et al. 2017). Then the Basic Local 170 Alignment Search Tool (BLAST) was applied to screen mRNAs that have complementary 171 sequences to lncRNAs, followed by RNAplex (Liang et al. 2017) to identify trans-regulated 172 target genes of lncRNAs. Subsequently, DELs and their corresponding target DEGs were 173 obtained.

\section{Prediction of microRNAs both targeted to key DELs and DEGs}

175 We predicted the target microRNAs (miRNAs) of DELs and DEGs in key lncRNA-mRNA 176 pairs. TargetScan (Agarwal et al. 2015) was carried out to predict the target miRNAs of key 177 DEGs, and the RNAhybrid (Rehmsmeier et al. 2004) was applied to predict the target miRNAs 178 of key DELs.

\section{Construction of interaction network}

180

Cytoscape version 3.6.1 was exerted to construct the networks of lncRNA-mRNA pairs and 181 182 183

protein-protein interaction networks with those downloaded files including connected nodes with confidence score.

\section{Statistical analysis}

All data were expressed as mean \pm standard deviation (SD) unless otherwise noted. The significant difference was measured using a two-tailed student t-test. $P<0.05$ was considered statistically significant. 
187 Results

188

189

190

191

192

193

194

195

196

197

198

199

200

201

202

203

204

205

206

207

208

209

210

211

\section{The characteristics of rats}

The plasma glucose concentration of GK rats was significantly higher than that of control

Wistar rats at 3 and 4 weeks of age $(P<0.001$, Table 1$)$, which was in accordance with previous research (Ando et al. 2018). Besides, the plasma glucose concentration of 3-week-old GK rats was significantly higher than that of 4 -week-old GK rats $(P<0.001$, Table 1$)$.

\section{Differentially expressed lncRNAs and $m R N A s$}

In total, we got 438 and 1000 differentially expressed mRNAs (DEGs) between GK and Wistar rats at 3 and 4 weeks of age, respectively (false discovery rate, FDR < 0.05) (Fig. 2A, Table S3). There were 401 and 746 differentially expressed lncRNAs (DELs) in GK rats compared with Wistar rats at 3 and 4 weeks of age, respectively (FDR < 0.05) (Fig. 2B, Table S4). Among the DEGs, 141 overlapping upregulated mRNAs and 103 overlapping downregulated mRNAs were detected between 3 and 4 weeks (Fig. 2A). A total of 91 overlapping upregulated lncRNAs and 124 overlapping downregulated lncRNAs were found between 3 and 4 weeks (Fig. 2B). From the results of enrichment pathway analysis of DEGs at 3 and 4 weeks, we found the insulin resistance pathway was the only one overlapping pathway between 3 and 4 weeks among top 10 KEGG pathways (Fig. 2C and 2D). The DEGs enriched in insulin resistance pathway were sterol regulatory element binding transcription factor 1 (Srebfl, also known as Srebp1c), solute carrier family 27 member 1 (Slc27a1), protein kinase C, theta (Prkcq), cAMP responsive element binding protein 3-like 1 (Creb3l1), forkhead box O1 (Foxo1), TBC1 domain family, member 4 (Tbcld4, also termed as AS160), and carnitine palmitoyltransferase 1A (Cpt1a).

To obtain the interaction of the proteins encoded by 141 overlapping upregulated genes and 103 overlapping downregulated genes between 3 and 4 weeks, we analyzed protein-protein interaction of these proteins using STRING. Next, we constructed the protein-protein interaction 
212 network (Fig. 3A and 3B). Then, the top 10 mRNAs according to the node degree among

213 network and their corresponding node degrees were listed in Table S5. The network among top

21410 upregulated and downregulated node mRNAs were shown in Fig. 3C and 3D. The

215 dysregulated genes Srebf1, Slc27a1, Foxo1 and Cpt1a that enriched in insulin resistance pathway 216 also existed in the network of top 10 upregulated and downregulated node mRNAs (Fig. 3C and

217 3D), which indicating that these four genes might be important for the development of

218 hyperglycemia and T2D in GK rats at the age of 3 and 4 weeks.

219

220

221

222

223

224

225

226

227

228

229

230

231

232

233

234

235

236

The co-expressed lncRNA-mRNA pairs with high correlation coefficients

To investigate the potential function of these DELs, we performed lncRNA-mRNA coexpression network analysis. After filtering, a total of 901 co-expressed lncRNA-mRNA pairs with high correlation coefficients were selected, including 136 DEGs and 120 DELs (Table S6). 136 DEGs were enriched in two KEGG pathways $(P<0.05)$, including transcriptional misregulation in cancer and pathways in cancer. But both pathways were not related to T2D. 136 DEGs were enriched in biological processes (Fig. 4A). Among these DEGs, 2 DEGs (pyruvate dehydrogenase kinase 4, Pdk4 and Cptla) were enriched in "regulation of fatty acid oxidation", and 3 DEGs (Foxo1, Pdk4 and Sh2b2) were enriched in "insulin receptor signaling pathway". The top 10 nodes ranked by degrees in co-expressed lncRNA-mRNA network were consist of 7 mRNAs and 3 lncRNAs (Table 2). The network of these dysregulated mRNAs (Cep19, Cpt1a, Ephx2, Foxo1, Pdk4, Sh2b2 and Stc2) and their co-expressed lncRNAs were shown in Fig. 4B. And the expression of key co-expressed lncRNA-mRNA pairs were shown in Fig. 4C and 4D. Notably, the dysregulated genes Cptla, Foxol and Pdk4 also appeared in the network of top 10 upregulated and downregulated mRNAs (Fig. 4C and 4D), indicating that these mRNAs might associate with hyperglycemia and T2D in GK rats at the age of 3 and 4 weeks.

However, other genes involved in fatty acid transport and $\beta$-oxidation were not significantly dysregulated in GK rats at the age of 3 and 4 weeks (Table S7). In our study, genes related to 
237 glycolysis and glycogen synthesis were not dysregulated in GK rats at 3 and 4 weeks of age 238 (Table S7).

239 The predicated target $m R N A s$ of differentially expressed lncRNAs

240 To identify the potential role of dysregulated lncRNAs in the development of

241 hyperglycemia and T2D in GK rats at the age of 3 and 4 weeks, we predicted their cis- and trans-

242 target mRNAs. A total of 15 predicted cis-target DEGs and 88 predicted trans-target DEGs were 243 obtained in DELs at 3 weeks (Table S8). There were 31 predicted cis-target DEGs and 382

244 predicted trans-target DEGs in DELs at 4 weeks (Table S9). There were 32 overlapping DEL245 target DEGs between 3 and 4 weeks (Fig. 5A, Table S10). Network analysis for these 246 overlapping DEGs found out 32 lncRNA-mRNA pairs, including 18 DEGs and 19 DELs (Fig. 247 5B). Among the 18 DEGs, interleukin 15 (Ill5), F-box and WD repeat domain containing 7 $248(F b x w 7)$ and uncoupling protein $3(U c p 3)$ were related to increased glycaemia (Gray \& Kamolrat 249 2011; Zhao et al. 2018), glucose intolerance (Fujimoto et al. 2019), and increased fatty acid 250 oxidation (Bezaire et al. 2005). Among those 32 lncRNA-target mRNA pairs, 5 lncRNA-target 251 mRNA pairs (NONRATG014028.2-Pim1, NONRATG011882.2-Il15, NONRATG013497.2-

252 Fbxw7, NONRATG011747.2-Mrps35, and MSTRG.1662-Ucp3) appeared in 901 co-expressed 253 lncRNA-mRNA pairs with high correlation coefficients, suggesting these dysregulated lncRNA254 mRNA pairs might involve in the hyperglycemia and T2D of GK rats at the age of 3 and 4 255 weeks. And the relative expression of NONRATG011882.2-Il15, NONRATG013497.2-Fbxw7, 256 and MSTRG.1662-Ucp3 pairs were shown in Fig. 5C.

257 The target microRNAs (miRNAs) of key DEGs and DELs

258 To explore the role of IncRNAs in the expression of mRNAs, we predicted the target 259 miRNA of key DELs and DEGs. Then we obtained the overlapping miRNAs targeted both 260 DEGs and DELs in key lncRNA-mRNA pairs (Fig. 6), which provided miRNAs linkers between 261 DELs and DEGs. We found that rno-miR-139-5p, rno-miR-486 and rno-miR-93-5p target both 
262 MSTRG.14356 and Foxol (Fig. 6). We got three target miRNAs rno-miR-20b-5p, rno-miR-27a-

$2633 \mathrm{p}$ and rno-miR-17-5p of MSTRG.2584 and Foxol (Fig. 6). The target miRNAs of Stc2 and

264 MSTRG.2584 were rno-miR-24-3p, rno-miR-532-5p, rno-miR-181a-5p and rno-miR-181b-5p

265 (Fig. 6). MiRNAs rno-miR-195-5p, rno-miR-181b-5p, rno-miR-23b-3p, rno-miR-139-5p and

266 rno-miR-23a-3p were the target miRNAs of MSTRG (Fig. 6).12678 and Pdk4. Three miRNAs

267 including rno-miR-34a-5p, rno-miR-125a-5p and rno-miR-125b-5p targeted MSTRG.1662 and

$268 U c p 3$ (Fig. 6). Additionally, the target miRNA of NONRATG013497.2 and Fbxw7 was rno-

269 miR-24-3p (Fig. 6). And rno-miR-326-3p was the target of NONRATG011882.2 and Il15 (Fig.

$2706)$.

271 Discussion

272 In the present study, we obtained mRNA and lncRNA expression profiles of skeletal muscle

273 of GK and Wistar rats at 3 and 4 weeks of age by RNA-sequencing. In total, 438 DEGs and 401

274 DELs were obtained in skeletal muscle of GK rats compared with Wistar rats at the age of 3

275 weeks (FDR < 0.05), 1000 DEGs and 746 DELs at 4 weeks of age (FDR $<0.05)$. To address the

276 function of those DELs, we screened the co-expressed lncRNA-mRNA pairs with high

277 correlation coefficients, predicted the target mRNAs of DELs and predicted miRNAs targeted

278 both DEGs and DELs. In considering previous studies, our results indicated that the dysregulated

279 expressed lncRNA-mRNA pairs might be implicated in hyperglycemia, glucose intolerance, and

280 increased fatty acid oxidation in GK rats at the age of 3 and 4 weeks. However, the annotation of

281 IncRNAs is incomplete, and the function of them has not been explained clearly. Thereby,

282 further studies are necessary to reveal their potential function.

283 The DEGs Slc27a1, Cpt1a, Srebf1, and Foxo1 were enriched in insulin resistance pathway

284 and also appeared in the network of top 10 upregulated and downregulated mRNAs, indicating

285 these four mRNAs might play key roles in the development of hyperglycemia and T2D in GK

286 rats at the age of 3 and 4 weeks. It has been demonstrated that SLC27A1 was implicated in the

287 regulation of fatty acid transport and oxidation. Overexpression of Slc27al could increase fatty

Peer) reviewing PDF | (2019:10:42596:1:0:NEW 3 Jan 2020) 
288 acid uptake and oxidation in L6E9 skeletal muscle cells (Sebastian et al. 2009). CPT1A encoded 289 by Cpt1a is responsible for transport long-chain fatty acid into mitochondria. And

290 overexpression of Cpt1a could lead to enhanced fatty acid oxidation in hepatocytes, $\beta$-Cells and 291 muscle cells (Akkaoui et al. 2009; Herrero et al. 2005; Perdomo et al. 2004; Stefanovic-Racic et 292 al. 2008). Srebf1 is a transcription factor regulating fatty acid synthesis. The $o b / o b$ mice with 293 inactivated SREBF1 showed reduced hepatic fatty acid synthesis (Moon et al. 2012). Blood 294 glucose was significantly higher in $S r e b f 1^{-/-}$mice than in $S r e b f 1^{+/+}$mice (Jang et al. 2016). It has 295 been unveiled that inhibiting expression of Foxol could increase glucose oxidation in mouse 296 heart (Gopal et al. 2017). Moreover, TBC1D4 was reported to be involved in glucose uptake. 297 Whole-body knockout Tbcld4 mice exhibited markedly decreased insulin-stimulated glucose 298 uptake in skeletal muscle (Lansey et al. 2012; Wang et al. 2013a). Therefore, in our study, the 299 significantly increased expression of Slc27al, Cptla and Foxol might associate with increased 300 fatty acid transport and oxidation in skeletal muscle of GK rats at 3 and 4 weeks of age. Randle 301 et al pointed out, increased oxidation of fatty acids could repress glucose oxidation (Hue \&

302 Taegtmeyer 2009; Randle et al. 1963). Thus, increased fatty acid transport and oxidation might 303 be related to increased glucose concentration in GK rats at the age of 3 and 4 weeks. 304 Additionally, the significantly reduced expression of Tbcld4 might be related to decreased 305 glucose uptake in skeletal muscle of GK rats at 3 and 4 weeks of age. Taken together, our results 306 indicated that Srebfl, Slc27a1, Foxo1, Tbc1d4, and Cptla might be related to increased

307 glycaemia in GK rats at 3 and 4 weeks of age.

308 As GK rats are produced from Wistar rats with impaired glucose tolerance, which plays an 309 essential role in T2D development of GK rats. In this study, Ephx2, Stc2, Cep19, Il15 and Fbxw7 310 genes were found to be associated with impaired glucose tolerance and hyperglycemia. Elevated

311 epoxyeicosatrienoic acids, which was inhibited by the enzyme encoded by Ephx2, could improve 312 insulin-stimulated glucose uptake in skeletal muscle of $d b / d b$ mice (Shim et al. 2014).

313 Additionally, previous studies showed that mice with whole-body knockout Ephx2 exhibited 314 improved insulin secretion (Luo et al. 2010; Luria et al. 2011). Moreover, plasma glucose 
315 clearance was faster in whole-body knockout Ephx2 mice than that in wild-type mice (Luria et 316 al. 2011). As the expression of Ephx2 was lower in skeletal muscle than in kidney and liver, the 317 reduced Ephx2 might have a weaker effect on glucose clearance in skeletal muscle of GK rats at 318 the age of 3 and 4 weeks. Whole-body Stc2 and Cep19 knockout mice displayed significantly 319 increased circulating glucose concentration (Lopez et al. 2018), and markedly impaired glucose 320 tolerance and insulin resistant (Shalata et al. 2013), respectively. It has been explored that

321 overexpressed Il15 transgenic mice showed better glucose tolerance compared to wild-type mice, 322 and Glut4 translocation was promoted in skeletal muscle by AMP-Activated protein kinase 323 pathway (Fujimoto et al. 2019). In addition, skeletal muscle-specific overexpression of Il15 324 transgenic mice displayed greater insulin sensitivity and decreased glucose concentration (Quinn 325 et al. 2011). Liver-specific Fbxw7 knockout mice presented hyperglycemia, glucose intolerance, 326 and insulin resistance (Zhao et al. 2018). Thus, the significantly downregulated Stc2, Il15, and 327 Fbxw7 might associate with hyperglycemia and impaired glucose tolerance in GK rats at 3 and 4 328 weeks of age. Since targeted lncRNAs of these significantly downregulated mRNAs had a high 329 correlation coefficient, the co-expressed pairs lncRNA-mRNA pairs, such as 330 NONRATG003318.2-Stc2, NONRATG011882.2-Il15, and NONRATG013497.2-Fbxw7 be 331 related to hyperglycemia and impaired glucose tolerance in GK rats at the age of 3 and 4 weeks. It is well known that increased fatty acid oxidation could inhibit glucose oxidation in heart 333 and skeletal muscle (Hue \& Taegtmeyer 2009; Randle et al. 1963). Thus, the dysregulated fatty 334 acid oxidation might affect circulating glucose concentration. Pdk4 and Ucp3 were found to be associated with increased fatty acid oxidation. Upregulated Pdk4 could decrease glucose oxidation and enhance fatty acid oxidation in myocardium and skeletal muscle (Sugden \&

337 Holness 2003; Zhao et al. 2008). As one transcriptional factor, Foxol could regulate the 338 expression of $P d k 4$, and the inhibition of it could increase glucose oxidation in mouse heart 339 (Gopal et al. 2017). Ucp3, located in mitochondrial inner membrane, expressed predominantly of 340 skeletal muscle in humans and rodents (Boss et al. 1997). Whole-body Ucp3 overexpression 341 mice showed increased activity of enzymes that implicated in fatty acid oxidation in skeletal 
342 muscle (Bezaire et al. 2005). The significantly decreased rate of long-chain fatty acid oxidation

343 was observed in rat heart with partial loss of $U c p 3$ gene $\left(U c p 3^{+/-}\right)$(Edwards et al. 2018). Hence,

344 in our study, the significantly increased $P d k 4$, and $U c p 3$ and their corresponding co-expressed or

345 targeted lncRNAs, including NONRATG017315.2-Pdk4, and MSTRG.1662-Ucp3 might

346 contribute to increased glycaemia and increased fatty acid oxidation in GK rats at 3 and 4 weeks

347 of age.

348 Glycolysis and glycogen synthesis were demonstrated to be involved in glucose

349 homeostasis (Hwang et al. 1995; Rothman et al. 1992; Shulman et al. 1990). However, in our

350 study, genes related to glycolysis and glycogen synthase were not dysregulated in GK rats at the 351 age of 3 and 4 weeks.

352 Recently, evidences showed that lncRNAs could regulate mRNAs by interacting with 353 microRNAs (miRNAs) (Zhang \& Zhu 2014). Among the target miRNA of mRNAs we 354 predicted, miR-139 has been identified could target Foxol directly and inhibit its expression in 355 mice hepatocytes (Hasseine et al. 2009). What's more, miR-139 overexpression leads to 356 markedly reduced Foxol level, and the inhibition of miR-139 contribute to increased Foxol level 357 (Yan et al. 2018). Moreover, Foxol was the target gene of miR-486-5p (Liu et al. 2019). Thus, 358 the DELs in key lncRNA-mRNA pairs might regulate mRNAs level through binding to their 359 common miRNAs. Further studies are needed to identify the target miRNAs of DEGs and DELs 360 and measure the miRNAs profiles in skeletal muscle of GK rats in the future.

\section{Conclusions}

In the present study, we found that the dysregulated lncARNA-mRNA pairs NONRATG013497.2-Fbxw7 and MSTRG.1662-Ucp3) might be implicated in hyperglycemia, glucose intolerance, as well as dysregulated glucose and fatty acid oxidation in GK rats at 3 and 4 weeks of age. These results may provide more comprehensive knowledge about mRNAs and 
368 may serve as important resources for future studies to investigate the regulatory mechanism of

lncRNAs in skeletal muscle of GK rats at the age of 3 and 4 weeks.

370

371

372

373

374

375

376

377

378

379

380

381

382

383

384

385

386

387

388

389

390

391

392

393

394

395

396

397

398

399

400

401

402

403

404

Agarwal V, Bell GW, Nam JW, and Bartel DP. 2015. Predicting effective microRNA target sites in mammalian mRNAs. Elife 4. 10.7554/eLife.05005

Akerman I, Tu Z, Beucher A, Rolando DMY, Sauty-Colace C, Benazra M, Nakic N, Yang J, Wang H, Pasquali L, Moran I, Garcia-Hurtado J, Castro N, Gonzalez-Franco R, Stewart AF, Bonner C, Piemonti L, Berney T, Groop L, Kerr-Conte J, Pattou F, Argmann C, Schadt E, Ravassard P, and Ferrer J. 2017. Human Pancreatic beta Cell IncRNAs Control Cell-Specific Regulatory Networks. Cell Metab 25:400-411. 10.1016/j.cmet.2016.11.016

Akkaoui M, Cohen I, Esnous C, Lenoir V, Sournac M, Girard J, and Prip-Buus C. 2009. Modulation of the hepatic malonyl-CoA-carnitine palmitoyltransferase $1 \mathrm{~A}$ partnership creates a metabolic switch allowing oxidation of de novo fatty acids. Biochem J 420:429-438. 10.1042/BJ20081932

Almon RR, Dubois DC, Sukumaran S, Wang X, Xue B, Nie J, and Jusko WJ. 2012. Effects of high fat feeding on liver gene expression in diabetic goto-kakizaki rats. Gene Regul Syst Bio 6:151-168. 10.4137/GRSB.S10371

Ando A, Gantulga D, Nakata M, Maekawa F, Dezaki K, Ishibashi S, and Yada T. 2018. Weaning stage hyperglycemia induces glucose-insensitivity in arcuate POMC neurons and hyperphagia in type 2 diabetic GK rats. Neuropeptides 68:49-56. 10.1016/j.npep.2018.02.001

Baron AD, Brechtel G, Wallace P, and Edelman SV. 1988. Rates and tissue sites of non-insulin- and insulin-mediated glucose uptake in humans. Am J Physiol 255:E769-774. 10.1152/ajpendo.1988.255.6.E769

Bezaire V, Spriet LL, Campbell S, Sabet N, Gerrits M, Bonen A, and Harper ME. 2005. Constitutive UCP3 overexpression at physiological levels increases mouse skeletal muscle capacity for fatty acid transport and oxidation. FASEB J 19:977-979. 10.1096/fj.04-2765fje

Bisbis S, Bailbe D, Tormo MA, Picarel-Blanchot F, Derouet M, Simon J, and Portha B. 1993. Insulin resistance in the GK rat: decreased receptor number but normal kinase activity in liver. Am J Physiol 265:E807-813. 10.1152/ajpendo.1993.265.5.E807

Boss O, Samec S, Paoloni-Giacobino A, Rossier C, Dulloo A, Seydoux J, Muzzin P, and Giacobino JP. 1997. Uncoupling protein-3: a new member of the mitochondrial carrier family with tissue-specific expression. FEBS Lett 408:39-42.

Cabili MN, Trapnell C, Goff L, Koziol M, Tazon-Vega B, Regev A, and Rinn JL. 2011. Integrative annotation of human large intergenic noncoding RNAs reveals global properties and specific subclasses. Genes Dev 25:19151927. 10.1101/gad.17446611

Dadke SS, Li HC, Kusari AB, Begum N, and Kusari J. 2000. Elevated expression and activity of protein-tyrosine phosphatase 1B in skeletal muscle of insulin-resistant type II diabetic Goto-Kakizaki rats. Biochem Biophys Res Commun 274:583-589. 10.1006/bbrc.2000.3188

DeFronzo RA, Jacot E, Jequier E, Maeder E, Wahren J, and Felber JP. 1981. The effect of insulin on the disposal of intravenous glucose. Results from indirect calorimetry and hepatic and femoral venous catheterization. Diabetes 30:1000-1007.

PeerJ reviewing PDF | (2019:10:42596:1:0:NEW 3 Jan 2020) 
405

406

407

408

409

410

411

412

413

414

415

416

417

418

419

420

421

422

423

424

425

426

427

428

429

430

431

432

433

434

435

436

437

438

439

440

441

442

443

444

445

Derrien T, Johnson R, Bussotti G, Tanzer A, Djebali S, Tilgner H, Guernec G, Martin D, Merkel A, Knowles DG, Lagarde J, Veeravalli L, Ruan X, Ruan Y, Lassmann T, Carninci P, Brown JB, Lipovich L, Gonzalez JM, Thomas M, Davis CA, Shiekhattar R, Gingeras TR, Hubbard TJ, Notredame C, Harrow J, and Guigo R. 2012. The GENCODE 7 catalog of human long noncoding RNAs: analysis of their gene structure, evolution, and expression. Genome Res 22:1775-1789. 10.1101/gr.132159.111

Djebali S, Davis CA, Merkel A, Dobin A, Lassmann T, Mortazavi A, Tanzer A, Lagarde J, Lin W, Schlesinger F, Xue C, Marinov GK, Khatun J, Williams BA, Zaleski C, Rozowsky J, Roder M, Kokocinski F, Abdelhamid RF, Alioto T, Antoshechkin I, Baer MT, Bar NS, Batut P, Bell K, Bell I, Chakrabortty S, Chen X, Chrast J, Curado J, Derrien T, Drenkow J, Dumais E, Dumais J, Duttagupta R, Falconnet E, Fastuca M, Fejes-Toth K, Ferreira P, Foissac S, Fullwood MJ, Gao H, Gonzalez D, Gordon A, Gunawardena H, Howald C, Jha S, Johnson R, Kapranov P, King B, Kingswood C, Luo OJ, Park E, Persaud K, Preall JB, Ribeca P, Risk B, Robyr D, Sammeth M, Schaffer L, See LH, Shahab A, Skancke J, Suzuki AM, Takahashi H, Tilgner H, Trout D, Walters N, Wang H, Wrobel J, Yu Y, Ruan X, Hayashizaki Y, Harrow J, Gerstein M, Hubbard T, Reymond A, Antonarakis SE, Hannon G, Giddings MC, Ruan Y, Wold B, Carninci P, Guigo R, and Gingeras TR. 2012. Landscape of transcription in human cells. Nature 489:101-108. 10.1038/nature11233

Dobin A, and Gingeras TR. 2015. Mapping RNA-seq Reads with STAR. Curr Protoc Bioinformatics 51:11 14 11-19. 10.1002/0471250953.bi1114s51

Edwards KS, Ashraf S, Lomax TM, Wiseman JM, Hall ME, Gava FN, Hall JE, Hosler JP, and Harmancey R. 2018. Uncoupling protein 3 deficiency impairs myocardial fatty acid oxidation and contractile recovery following ischemia/reperfusion. Basic Res Cardiol 113:47. 10.1007/s00395-018-0707-9

Espinoza CA, Allen TA, Hieb AR, Kugel JF, and Goodrich JA. 2004. B2 RNA binds directly to RNA polymerase II to repress transcript synthesis. Nat Struct Mol Biol 11:822-829. 10.1038/nsmb812

Esteller M. 2011. Non-coding RNAs in human disease. Nat Rev Genet 12:861-874. 10.1038/nrg3074

Frazee AC, Pertea G, Jaffe AE, Langmead B, Salzberg SL, and Leek JT. 2015. Ballgown bridges the gap between transcriptome assembly and expression analysis. Nat Biotechnol 33:243-246. 10.1038/nbt.3172

Fujimoto T, Sugimoto K, Takahashi T, Yasunobe Y, Xie K, Tanaka M, Ohnishi Y, Yoshida S, Kurinami H, Akasaka H, Takami Y, Takeya Y, Yamamoto K, and Rakugi H. 2019. Overexpression of Interleukin-15 exhibits improved glucose tolerance and promotes GLUT4 translocation via AMP-Activated protein kinase pathway in skeletal muscle. Biochem Biophys Res Commun 509:994-1000. 10.1016/j.bbrc.2019.01.024

Gao Y, Wu F, Zhou J, Yan L, Jurczak MJ, Lee HY, Yang L, Mueller M, Zhou XB, Dandolo L, Szendroedi J, Roden M, Flannery C, Taylor H, Carmichael GG, Shulman GI, and Huang Y. 2014. The H19/let-7 double-negative feedback loop contributes to glucose metabolism in muscle cells. Nucleic Acids Res 42:13799-13811. 10.1093/nar/gku1160

Gopal K, Saleme B, Al Batran R, Aburasayn H, Eshreif A, Ho KL, Ma WK, Almutairi M, Eaton F, Gandhi M, Park EA, Sutendra G, and Ussher JR. 2017. FoxO1 regulates myocardial glucose oxidation rates via transcriptional control of pyruvate dehydrogenase kinase 4 expression. Am J Physiol Heart Circ Physiol 313:H479-H490. 10.1152/ajpheart.00191.2017

Goto Y, Kakizaki M, and Masaki N. 1976. Production of spontaneous diabetic rats by repetition of selective breeding. Tohoku J Exp Med 119:85-90.

Gray SR, and Kamolrat T. 2011. The effect of exercise induced cytokines on insulin stimulated glucose transport in C2C12 cells. Cytokine 55:221-228. 10.1016/j.cyto.2011.04.019 
446 Guil S, and Esteller M. 2012. Cis-acting noncoding RNAs: friends and foes. Nat Struct Mol Biol 19:1068-1075.

$447 \quad 10.1038 / \mathrm{nsmb} .2428$

448 Guttman M, and Rinn JL. 2012. Modular regulatory principles of large non-coding RNAs. Nature 482:339-346.

$449 \quad 10.1038 /$ nature10887

450 Hasseine LK, Hinault C, Lebrun P, Gautier N, Paul-Bellon R, and Van Obberghen E. 2009. miR-139 impacts FoxO1

451 action by decreasing FoxO1 protein in mouse hepatocytes. Biochem Biophys Res Commun 390:1278-1282.

$452 \quad$ 10.1016/j.bbrc.2009.10.135

453 Herrero L, Rubi B, Sebastian D, Serra D, Asins G, Maechler P, Prentki M, and Hegardt FG. 2005. Alteration of the

454 malonyl-CoA/carnitine palmitoyltransferase I interaction in the beta-cell impairs glucose-induced insulin

455 secretion. Diabetes 54:462-471.

456 Hue L, and Taegtmeyer H. 2009. The Randle cycle revisited: a new head for an old hat. Am J Physiol Endocrinol

457 Metab 297:E578-591. 10.1152/ajpendo.00093.2009

458 Hwang JH, Perseghin G, Rothman DL, Cline GW, Magnusson I, Petersen KF, and Shulman GI. 1995. Impaired net

459 hepatic glycogen synthesis in insulin-dependent diabetic subjects during mixed meal ingestion. A $13 \mathrm{C}$

$460 \quad$ nuclear magnetic resonance spectroscopy study. J Clin Invest 95:783-787. 10.1172/JCI117727

461 Jang H, Lee GY, Selby CP, Lee G, Jeon YG, Lee JH, Cheng KK, Titchenell P, Birnbaum MJ, Xu A, Sancar A, and Kim JB.

462 2016. SREBP1C-CRY1 signalling represses hepatic glucose production by promoting FOXO1 degradation

463

464 during refeeding. Nat Commun 7:12180. 10.1038/ncomms12180

Jia H, Osak M, Bogu GK, Stanton LW, Johnson R, and Lipovich L. 2010. Genome-wide computational identification

465

466

467

468

469

470

471

472

473

474

475

476

477

478

479

480

481

482

483

484

485 and manual annotation of human long noncoding RNA genes. RNA 16:1478-1487. 10.1261/rna.1951310

Kang YJ, Yang DC, Kong L, Hou M, Meng YQ, Wei L, and Gao G. 2017. CPC2: a fast and accurate coding potential calculator based on sequence intrinsic features. Nucleic Acids Res 45:W12-W16. 10.1093/nar/gkx428

Kayala MA, and Baldi P. 2012. Cyber-T web server: differential analysis of high-throughput data. Nucleic Acids Res 40:W553-559. 10.1093/nar/gks420

Kitahara A, Toyota T, Kakizaki M, and Goto Y. 1978. Activities of hepatic enzymes in spontaneous diabetes rats produced by selective breeding of normal Wistar rats. Tohoku J Exp Med 126:7-11.

Lansey MN, Walker NN, Hargett SR, Stevens JR, and Keller SR. 2012. Deletion of Rab GAP AS160 modifies glucose uptake and GLUT4 translocation in primary skeletal muscles and adipocytes and impairs glucose homeostasis. Am J Physiol Endocrinol Metab 303:E1273-1286. 10.1152/ajpendo.00316.2012

Liang Y, Yu B, Wang Y, Qiao Z, Cao T, and Zhang P. 2017. Duodenal long noncoding RNAs are associated with glycemic control after bariatric surgery in high-fat diet-induced diabetic mice. Surg Obes Relat Dis 13:1212-1226. 10.1016/j.soard.2017.02.010

Liu H, Ni Z, Shi L, Ma L, and Zhao J. 2019. MiR-486-5p inhibits the proliferation of leukemia cells and induces apoptosis through targeting FOXO1. Mol Cell Probes 44:37-43. 10.1016/j.mcp.2019.02.001

Liu JY, Yao J, Li XM, Song YC, Wang XQ, Li YJ, Yan B, and Jiang Q. 2014. Pathogenic role of IncRNA-MALAT1 in endothelial cell dysfunction in diabetes mellitus. Cell Death Dis 5:e1506. 10.1038/cddis.2014.466

Lopez JJ, Jardin I, Cantonero Chamorro C, Duran ML, Tarancon Rubio MJ, Reyes Panadero M, Jimenez F, Montero R, Gonzalez MJ, Martinez M, Hernandez MJ, Brull JM, Corbacho AJ, Delgado E, Granados MP, Gomez-Gordo L, Rosado JA, and Redondo PC. 2018. Involvement of stanniocalcins in the deregulation of glycaemia in obese mice and type 2 diabetic patients. J Cell Mol Med 22:684-694. 10.1111/jcmm.13355

Peer) reviewing PDF | (2019:10:42596:1:0:NEW 3 Jan 2020) 
486

487

488

489

490

491

492

493

494

495

496

497

498

499

500

501

502

503

504

505

506

507

508

509

510

511

512

513

514

515

516

517

518

519

520

521

522

523

524

525

Luo P, Chang HH, Zhou Y, Zhang S, Hwang SH, Morisseau C, Wang CY, Inscho EW, Hammock BD, and Wang MH. 2010. Inhibition or deletion of soluble epoxide hydrolase prevents hyperglycemia, promotes insulin secretion, and reduces islet apoptosis. J Pharmacol Exp Ther 334:430-438. 10.1124/jpet.110.167544

Luo Q, and Chen Y. 2016. Long noncoding RNAs and Alzheimer's disease. Clin Interv Aging 11:867-872. 10.2147/CIA.S107037

Luria A, Bettaieb A, Xi Y, Shieh GJ, Liu HC, Inoue H, Tsai HJ, Imig JD, Haj FG, and Hammock BD. 2011. Soluble epoxide hydrolase deficiency alters pancreatic islet size and improves glucose homeostasis in a model of insulin resistance. Proc Natl Acad Sci U S A 108:9038-9043. 10.1073/pnas.1103482108

Mercer TR, Dinger ME, Sunkin SM, Mehler MF, and Mattick JS. 2008. Specific expression of long noncoding RNAs in the mouse brain. Proc Natl Acad Sci U S A 105:716-721. 10.1073/pnas.0706729105

Moon YA, Liang G, Xie X, Frank-Kamenetsky M, Fitzgerald K, Koteliansky V, Brown MS, Goldstein JL, and Horton JD. 2012. The Scap/SREBP pathway is essential for developing diabetic fatty liver and carbohydrate-induced hypertriglyceridemia in animals. Cell Metab 15:240-246. 10.1016/j.cmet.2011.12.017

Moran I, Akerman I, van de Bunt M, Xie R, Benazra M, Nammo T, Arnes L, Nakic N, Garcia-Hurtado J, RodriguezSegui S, Pasquali L, Sauty-Colace C, Beucher A, Scharfmann R, van Arensbergen J, Johnson PR, Berry A, Lee C, Harkins T, Gmyr V, Pattou F, Kerr-Conte J, Piemonti L, Berney T, Hanley N, Gloyn AL, Sussel L, Langman L, Brayman KL, Sander M, McCarthy MI, Ravassard P, and Ferrer J. 2012. Human beta cell transcriptome analysis uncovers IncRNAs that are tissue-specific, dynamically regulated, and abnormally expressed in type 2 diabetes. Cell Metab 16:435-448. 10.1016/j.cmet.2012.08.010

Nie J, DuBois DC, Xue B, Jusko WJ, and Almon RR. 2017. Effects of High-Fat Feeding on Skeletal Muscle Gene Expression in Diabetic Goto-Kakizaki Rats. Gene Regul Syst Bio 11:1177625017710009. $10.1177 / 1177625017710009$

Nie J, Xue B, Sukumaran S, Jusko WJ, Dubois DC, and Almon RR. 2011. Differential muscle gene expression as a function of disease progression in Goto-Kakizaki diabetic rats. Mol Cell Endocrinol 338:10-17. 10.1016/j.mce.2011.02.016

Perdomo G, Commerford SR, Richard AM, Adams SH, Corkey BE, O'Doherty RM, and Brown NF. 2004. Increased beta-oxidation in muscle cells enhances insulin-stimulated glucose metabolism and protects against fatty acid-induced insulin resistance despite intramyocellular lipid accumulation. J Biol Chem 279:27177-27186. 10.1074/jbc.M403566200

Pertea M, Pertea GM, Antonescu CM, Chang TC, Mendell JT, and Salzberg SL. 2015. StringTie enables improved reconstruction of a transcriptome from RNA-seq reads. Nat Biotechnol 33:290-295. 10.1038/nbt.3122

Peterlin BM, Brogie JE, and Price DH. 2012. 7SK snRNA: a noncoding RNA that plays a major role in regulating eukaryotic transcription. Wiley Interdiscip Rev RNA 3:92-103. 10.1002/wrna.106

Portha B, Giroix MH, Tourrel-Cuzin C, Le-Stunff H, and Movassat J. 2012. The GK rat: a prototype for the study of non-overweight type 2 diabetes. Methods Mol Biol 933:125-159. 10.1007/978-1-62703-068-7_9

Quinn LS, Anderson BG, Conner JD, Pistilli EE, and Wolden-Hanson T. 2011. Overexpression of interleukin-15 in mice promotes resistance to diet-induced obesity, increased insulin sensitivity, and markers of oxidative skeletal muscle metabolism. Int J Interferon Cytokine Mediat Res 3:29-42. 10.2147/IJICMR.S19007

Randle PJ, Garland PB, Hales CN, and Newsholme EA. 1963. The glucose fatty-acid cycle. Its role in insulin sensitivity and the metabolic disturbances of diabetes mellitus. Lancet 1:785-789.

Peer] reviewing PDF | (2019:10:42596:1:0:NEW 3 Jan 2020) 
526

527

528

529

530

531

532

533

534

535

536

537

538

539

540

541

542

543

544

545

546

547

548

549

550

551

552

553

554

555

556

557

558

559

560

561

562

563

564

565

Reddy MA, Chen Z, Park JT, Wang M, Lanting L, Zhang Q, Bhatt K, Leung A, Wu X, Putta S, Saetrom P, Devaraj S, and Natarajan R. 2014. Regulation of inflammatory phenotype in macrophages by a diabetes-induced long noncoding RNA. Diabetes 63:4249-4261. 10.2337/db14-0298

Rehmsmeier M, Steffen P, Hochsmann M, and Giegerich R. 2004. Fast and effective prediction of microRNA/target duplexes. RNA 10:1507-1517. 10.1261/rna.5248604

Rinn JL, Kertesz M, Wang JK, Squazzo SL, Xu X, Brugmann SA, Goodnough LH, Helms JA, Farnham PJ, Segal E, and Chang HY. 2007. Functional demarcation of active and silent chromatin domains in human HOX loci by noncoding RNAs. Cell 129:1311-1323. 10.1016/j.cell.2007.05.022

Rothman DL, Shulman RG, and Shulman GI. 1992. 31P nuclear magnetic resonance measurements of muscle glucose-6-phosphate. Evidence for reduced insulin-dependent muscle glucose transport or phosphorylation activity in non-insulin-dependent diabetes mellitus. J Clin Invest 89:1069-1075. 10.1172/JCI115686

Sebastian D, Guitart M, Garcia-Martinez C, Mauvezin C, Orellana-Gavalda JM, Serra D, Gomez-Foix AM, Hegardt FG, and Asins G. 2009. Novel role of FATP1 in mitochondrial fatty acid oxidation in skeletal muscle cells. J Lipid Res 50:1789-1799. 10.1194/jlr.M800535-JLR200

Shalata A, Ramirez MC, Desnick RJ, Priedigkeit N, Buettner C, Lindtner C, Mahroum M, Abdul-Ghani M, Dong F, Arar N, Camacho-Vanegas O, Zhang R, Camacho SC, Chen Y, Ibdah M, DeFronzo R, Gillespie V, Kelley K, Dynlacht BD, Kim S, Glucksman MJ, Borochowitz ZU, and Martignetti JA. 2013. Morbid obesity resulting from inactivation of the ciliary protein CEP19 in humans and mice. Am J Hum Genet 93:1061-1071. 10.1016/j.ajhg.2013.10.025

Shim CY, Kim S, Chadderdon S, Wu M, Qi Y, Xie A, Alkayed NJ, Davidson BP, and Lindner JR. 2014. Epoxyeicosatrienoic acids mediate insulin-mediated augmentation in skeletal muscle perfusion and blood volume. Am J Physiol Endocrinol Metab 307:E1097-1104. 10.1152/ajpendo.00216.2014

Shulman GI, Rothman DL, Jue T, Stein P, DeFronzo RA, and Shulman RG. 1990. Quantitation of muscle glycogen synthesis in normal subjects and subjects with non-insulin-dependent diabetes by $13 \mathrm{C}$ nuclear magnetic resonance spectroscopy. N Engl J Med 322:223-228. 10.1056/NEJM199001253220403

Stefanovic-Racic M, Perdomo G, Mantell BS, Sipula IJ, Brown NF, and O'Doherty RM. 2008. A moderate increase in carnitine palmitoyltransferase 1 a activity is sufficient to substantially reduce hepatic triglyceride levels. Am J Physiol Endocrinol Metab 294:E969-977. 10.1152/ajpendo.00497.2007

Steiler TL, Galuska D, Leng Y, Chibalin AV, Gilbert M, and Zierath JR. 2003. Effect of hyperglycemia on signal transduction in skeletal muscle from diabetic Goto-Kakizaki rats. Endocrinology 144:5259-5267. 10.1210/en.2003-0447

Sugden MC, and Holness MJ. 2003. Recent advances in mechanisms regulating glucose oxidation at the level of the pyruvate dehydrogenase complex by PDKs. Am J Physiol Endocrinol Metab 284:E855-862. 10.1152/ajpendo.00526.2002

Sun L, Luo H, Bu D, Zhao G, Yu K, Zhang C, Liu Y, Chen R, and Zhao Y. 2013. Utilizing sequence intrinsic composition to classify protein-coding and long non-coding transcripts. Nucleic Acids Res 41:e166. 10.1093/nar/gkt646

Szklarczyk D, Franceschini A, Kuhn M, Simonovic M, Roth A, Minguez P, Doerks T, Stark M, Muller J, Bork P, Jensen $L$, and von Mering C. 2011. The STRING database in 2011: functional interaction networks of proteins, globally integrated and scored. Nucleic Acids Res 39:D561-568. 10.1093/nar/gkq973

PeerJ reviewing PDF | (2019:10:42596:1:0:NEW 3 Jan 2020) 
566 Tsoi LC, Iyer MK, Stuart PE, Swindell WR, Gudjonsson JE, Tejasvi T, Sarkar MK, Li B, Ding J, Voorhees JJ, Kang HM,

567

568

569

570

571

572

573

574

575

576

577

578

579

580

581

582

583

584

585

586

587

588

589

590

591

592

593

594

595

596
Nair RP, Chinnaiyan AM, Abecasis GR, and Elder JT. 2015. Analysis of long non-coding RNAs highlights tissue-specific expression patterns and epigenetic profiles in normal and psoriatic skin. Genome Biol 16:24. 10.1186/s13059-014-0570-4

Wang HY, Ducommun S, Quan C, Xie B, Li M, Wasserman DH, Sakamoto K, Mackintosh C, and Chen S. $2013 a$. AS160 deficiency causes whole-body insulin resistance via composite effects in multiple tissues. Biochem $J$ 449:479-489. 10.1042/BJ20120702

Wang L, Park HJ, Dasari S, Wang S, Kocher JP, and Li W. 2013b. CPAT: Coding-Potential Assessment Tool using an alignment-free logistic regression model. Nucleic Acids Res 41:e74. 10.1093/nar/gkt006

Xue B, Sukumaran S, Nie J, Jusko WJ, Dubois DC, and Almon RR. 2011. Adipose tissue deficiency and chronic inflammation in diabetic Goto-Kakizaki rats. PLoS One 6:e17386. 10.1371/journal.pone.0017386

Yan C, Li J, Feng S, Li Y, and Tan L. 2018. Long noncoding RNA Gomafu upregulates Foxo1 expression to promote hepatic insulin resistance by sponging miR-139-5p. Cell Death Dis 9:289. 10.1038/s41419-018-0321-7

Yin DD, Zhang EB, You LH, Wang N, Wang LT, Jin FY, Zhu YN, Cao LH, Yuan QX, De W, and Tang W. 2015. Downregulation of IncRNA TUG1 affects apoptosis and insulin secretion in mouse pancreatic beta cells. Cell Physiol Biochem 35:1892-1904. 10.1159/000373999

You L, Wang N, Yin D, Wang L, Jin F, Zhu Y, Yuan Q, and De W. 2016. Downregulation of Long Noncoding RNA Meg3 Affects Insulin Synthesis and Secretion in Mouse Pancreatic Beta Cells. J Cell Physiol 231:852-862. 10.1002/jcp.25175

Zhang $\mathrm{H}$, and Zhu JK. 2014. Emerging roles of RNA processing factors in regulating long non-coding RNAs. RNA Biol 11:793-797. 10.4161/rna.29731

Zhao G, Jeoung NH, Burgess SC, Rosaaen-Stowe KA, Inagaki T, Latif S, Shelton JM, McAnally J, Bassel-Duby R, Harris RA, Richardson JA, and Kliewer SA. 2008. Overexpression of pyruvate dehydrogenase kinase 4 in heart perturbs metabolism and exacerbates calcineurin-induced cardiomyopathy. Am J Physiol Heart Circ Physiol 294:H936-943. 10.1152/ajpheart.00870.2007

Zhao J, Xiong X, Li Y, Liu X, Wang T, Zhang H, Jiao Y, Jiang J, Zhang H, Tang Q, Gao X, Li X, Lu Y, Liu B, Hu C, and Li X. 2018. Hepatic F-Box Protein FBXW7 Maintains Glucose Homeostasis Through Degradation of Fetuin-A. Diabetes 67:818-830. 10.2337/db17-1348

Zhu X, Wu YB, Zhou J, and Kang DM. 2016. Upregulation of IncRNA MEG3 promotes hepatic insulin resistance via increasing FoxO1 expression. Biochem Biophys Res Commun 469:319-325. 10.1016/j.bbrc.2015.11.048 
Figure 1

Figure 1 Flowchart of data analysis pipeline.

FDR: false discovery rate. DEGs: differentially expressed mRNAs; DELs: differentially expressed IncRNAs; PPI: protein-protein interaction. GO: Gene Ontology; KEGG: Kyoto Encyclopedia of Genes and Genomes.

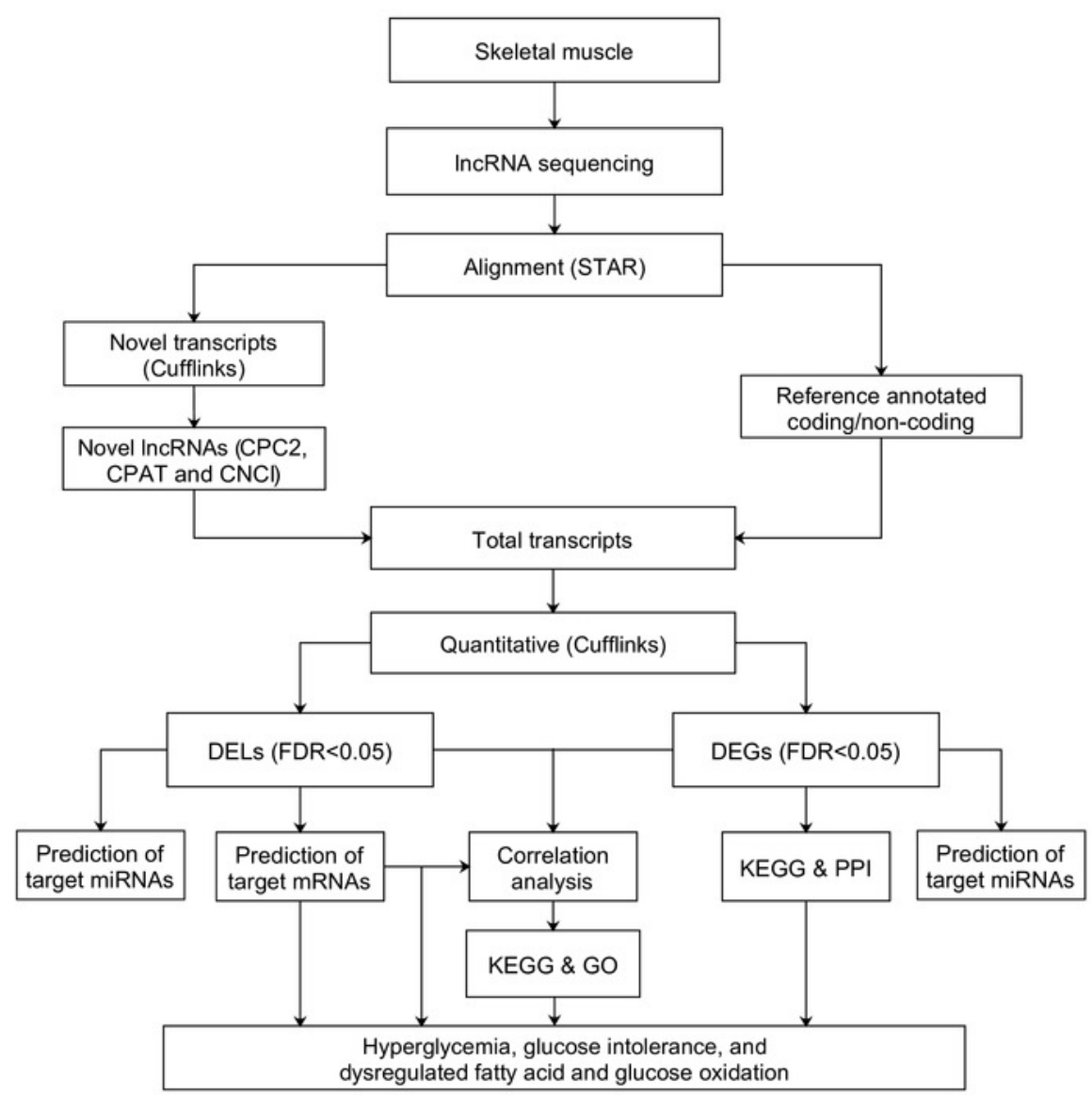




\section{Figure 2}

Figure 2 The differentially expressed IncRNAs and mRNAs in skeletal muscle of GK rats compared with aged-matched Wistar rats.

(A) Venn diagram of differentially expressed mRNAs (DEGs) in skeletal muscle of GK rats compared with aged-matched Wistar rats at the age of 3 and 4 weeks. (B) Venn diagram of differentially expressed IncRNAs (DELs) in skeletal muscle of GK rats compared with agedmatched Wistar rats at the age of 3 and 4 weeks. (C) The top 10 KEGG pathways of DEGs in GK rats compared with aged-matched Wistar rats at the age of 3 weeks. (D) The top 10 KEGG pathways of DEGs in GK rats compared with aged-matched Wistar rats at the age of 4 weeks. The 3wk_up_DEGs represents upregulated mRNAs at 3 weeks. The 4wk_up_DEGs represents upregulated mRNAs at 4 weeks. The 3wk_down_DEGs represents downregulated mRNAs at 3 weeks. The 4wk_down_DEGs represents downregulated mRNAs at 4 weeks. The 3wk_up_DELs represents upregulated IncRNAs at 3 weeks. The 4wk_up_DELs represents upregulated IncRNAs at 4 weeks. The 3wk_down_DELs represents downregulated IncRNAs at 3 weeks. The 4 wk_down_DELs represents downregulated IncRNAs at 4 weeks. 
A

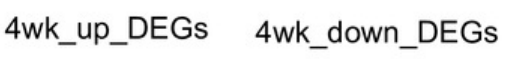

468

141

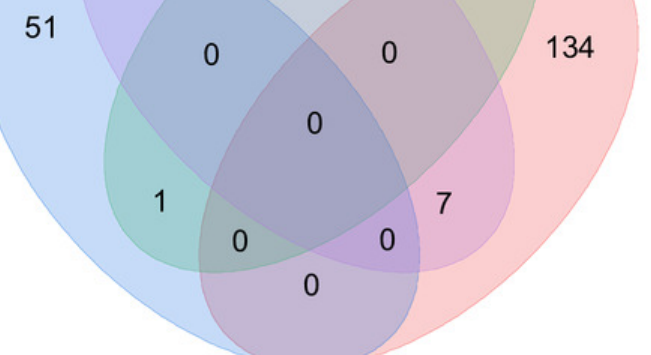

3wk_up_DEGs

3wk_down_DEGs

C

Top 10 KEGG pathways at 3 weeks

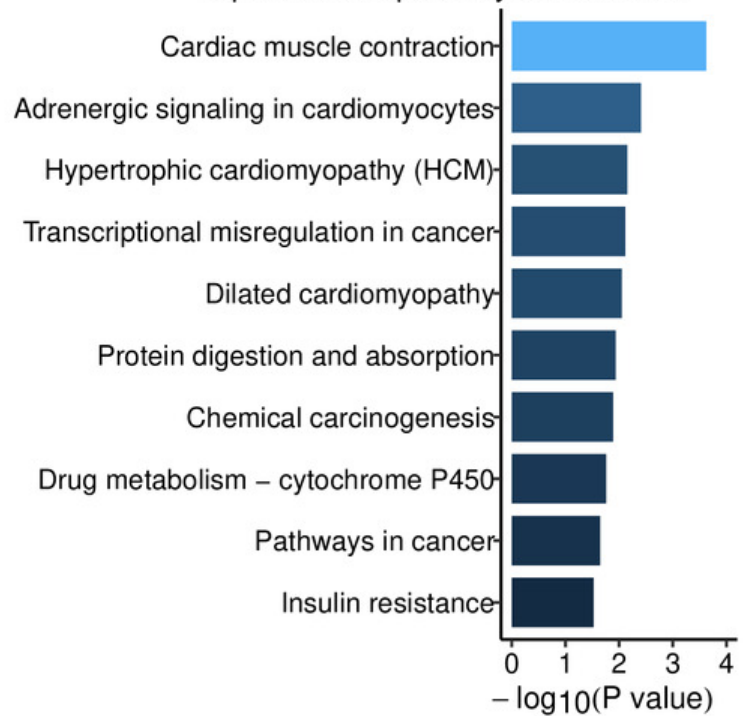

B 4wk_up_DELs 4wk_down_DELs

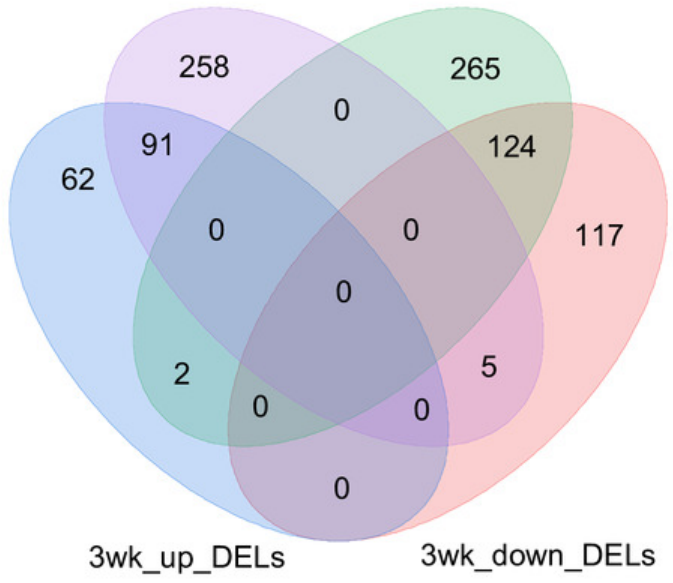

D Top $10 \mathrm{KEGG}$ pathways at 4 weeks

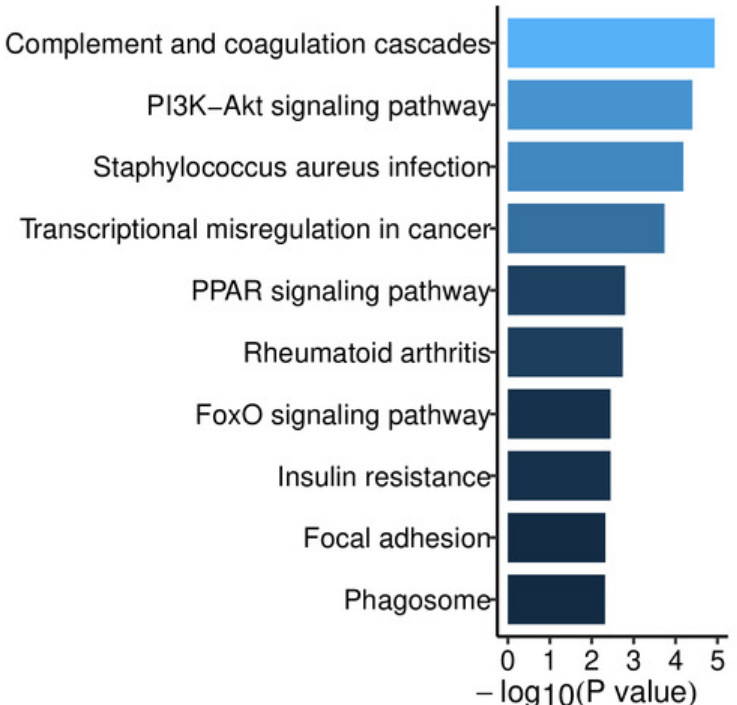




\section{Figure 3}

Figure 3 The top 10 mRNAs identified in protein-protein interaction networks.

(A) The protein-protein interaction network of overlapping upregulated DEGs at 3 and 4 weeks. The red represents significantly upregulated mRNAs in GK rats at the age of 3 and 4 weeks. (B) The protein-protein interaction network of overlapping downregulated DEGs at 3 and 4 weeks. The blue represents significantly downregulated mRNAs in GK rats at the age of 3 and 4 weeks. (C) The top 10 upregulated mRNAs ranked by node degree. The darker of the color indicates the higher of connectivity degree. (D) The top 10 downregulated mRNAs ranked by node degree. The darker of the color indicates the higher of connectivity degree. 
A
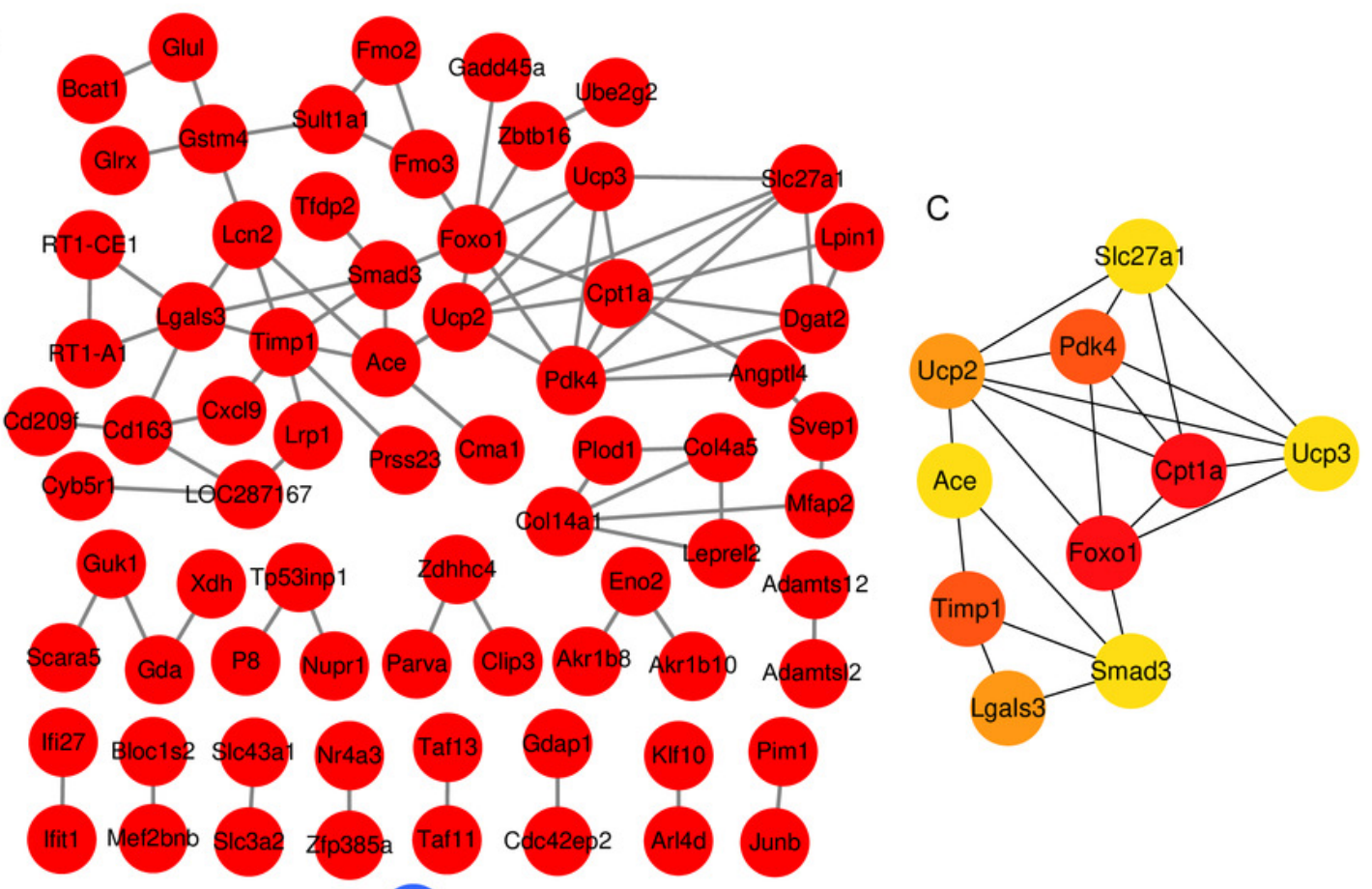

B
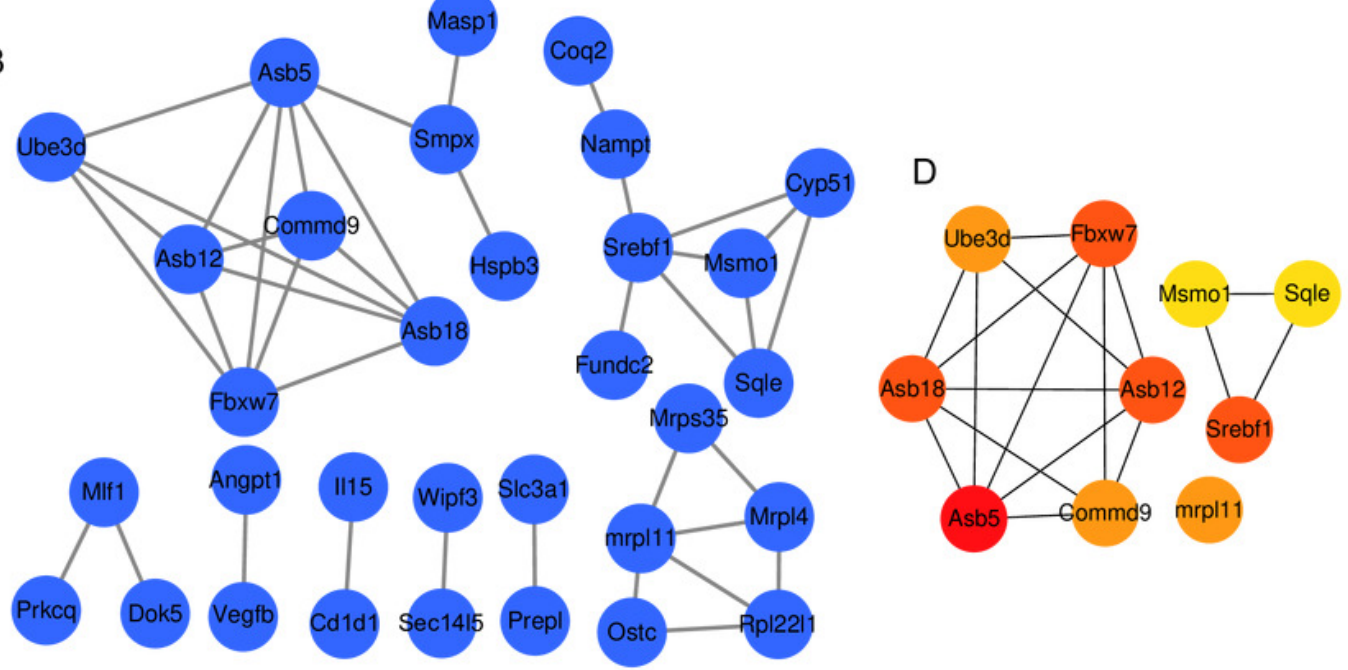


\section{Figure 4}

Figure 4 The co-expressed IncRNAs-mRNAs pairs with high correlation coefficients.

(A) The biological process of dysregulated mRNAs in co-expressed IncRNAs-mRNAs pairs with high correlation coefficients. The GO terms of biological process were as follows : GO: 0055114 oxidation-reduction process; GO: 0009267 cellular response to starvation; GO: 0050776 regulation of immune response; GO: 0017144 drug metabolic process; GO: 0048701 embryonic cranial skeleton morphogenesis; GO: 0034097 response to cytokine; GO: 0006955 immune response; GO: 0046320 regulation of fatty acid oxidation; GO: 0030091 protein repair; GO: 0006749 glutathione metabolic process; GO: 0007568 aging; GO: 0043434 response to peptide hormone; GO 0008286 insulin receptor signaling pathway; GO: 0006739 NADP metabolic process; GO 0006355 regulation of transcription, DNA-templated; GO 0032870 cellular response to hormone stimulus. (B) The network of key genes and their co-expressed IncRNAs. The red represents upregulated gene in GK rats compared with agedmatched Wistar rats at the age of 3 and 4 weeks. The blue represents downregulated gene in GK rats compared with aged-matched Wistar rats at the age of 3 and 4 weeks. The diamond represents IncRNA, while the circle represents mRNA. The line between IncRNA and mRNA represents the co-expression coefficient. The range of correlation coefficients was from 0.9 to 0.993573997. (C). The relative expression of Ephx2, Pdk4 and Stc2. (D) The relative expression of NONRATG017315.2, NONRATG010201.2, NONRATG011747.2, and NONRATG003318.2. Values are means $\pm \mathrm{SD}, * P<0.05, * * P<0.01$, $* * * P<0.001$ vs agematched Wistar group, $\mathrm{n}=6$. 


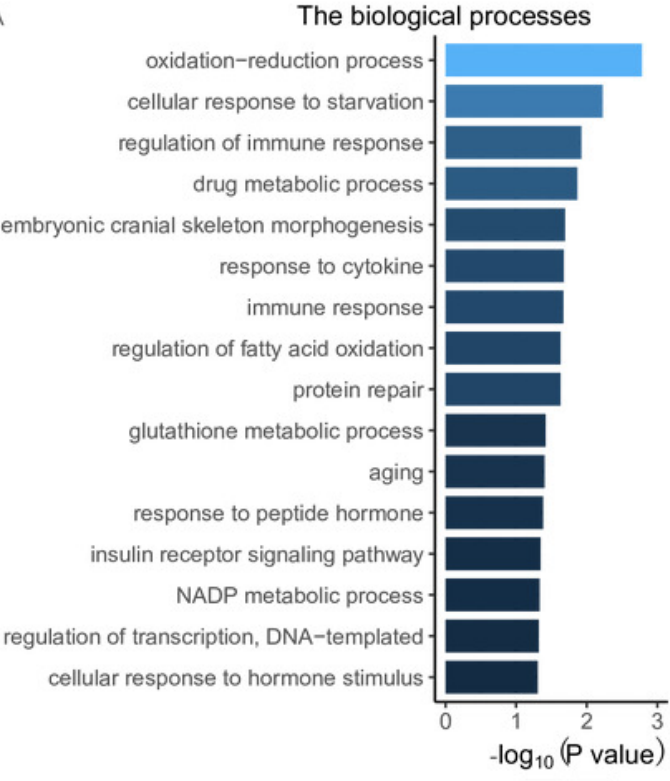

c

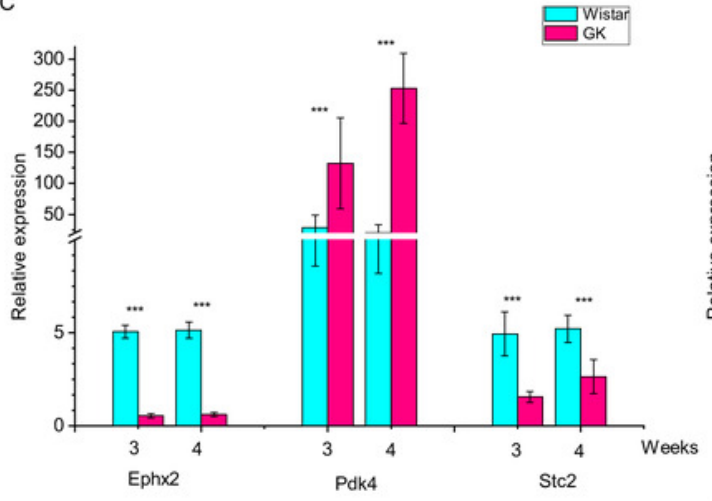

B
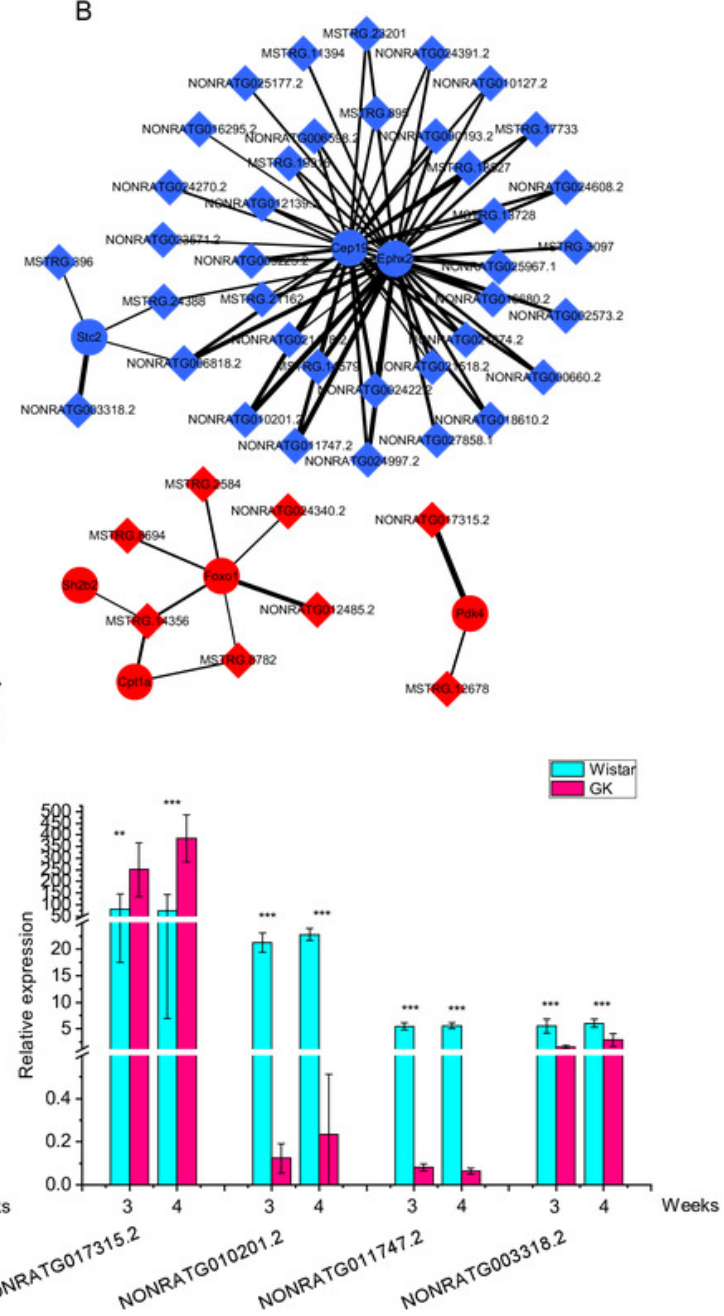


\section{Figure 5}

Figure 5 The differentially expressed target mRNAs for differentially expressed IncRNAs.

(A) Venn diagram of IncRNA-target mRNAs at 3 and 4 weeks. The DELs_target DEGs

represents the target differentially expressed mRNAs of differentially expressed IncRNAs. (B)

The network of overlapping IncRNA-target mRNAs at 3 and 4 weeks. The blue represents downregulated expression in GK rats compared with aged-matched Wistar rats at 3 and 4 weeks. The diamond represents IncRNA, the circle represents mRNA. (C) The relative expression of NONRATG011882.2-II15, NONRATG013497.2-Fbxw7, and MSTRG.1662-Ucp3. Values are means $\pm \mathrm{SD}, * P<0.05, * * P<0.01, * * * P<0.001$ vs age-matched Wistar group, $\mathrm{n}=6$. The red represents upregulated expression in GK rats compared with aged-matched Wistar rats at the age of 3 and 4 weeks. 


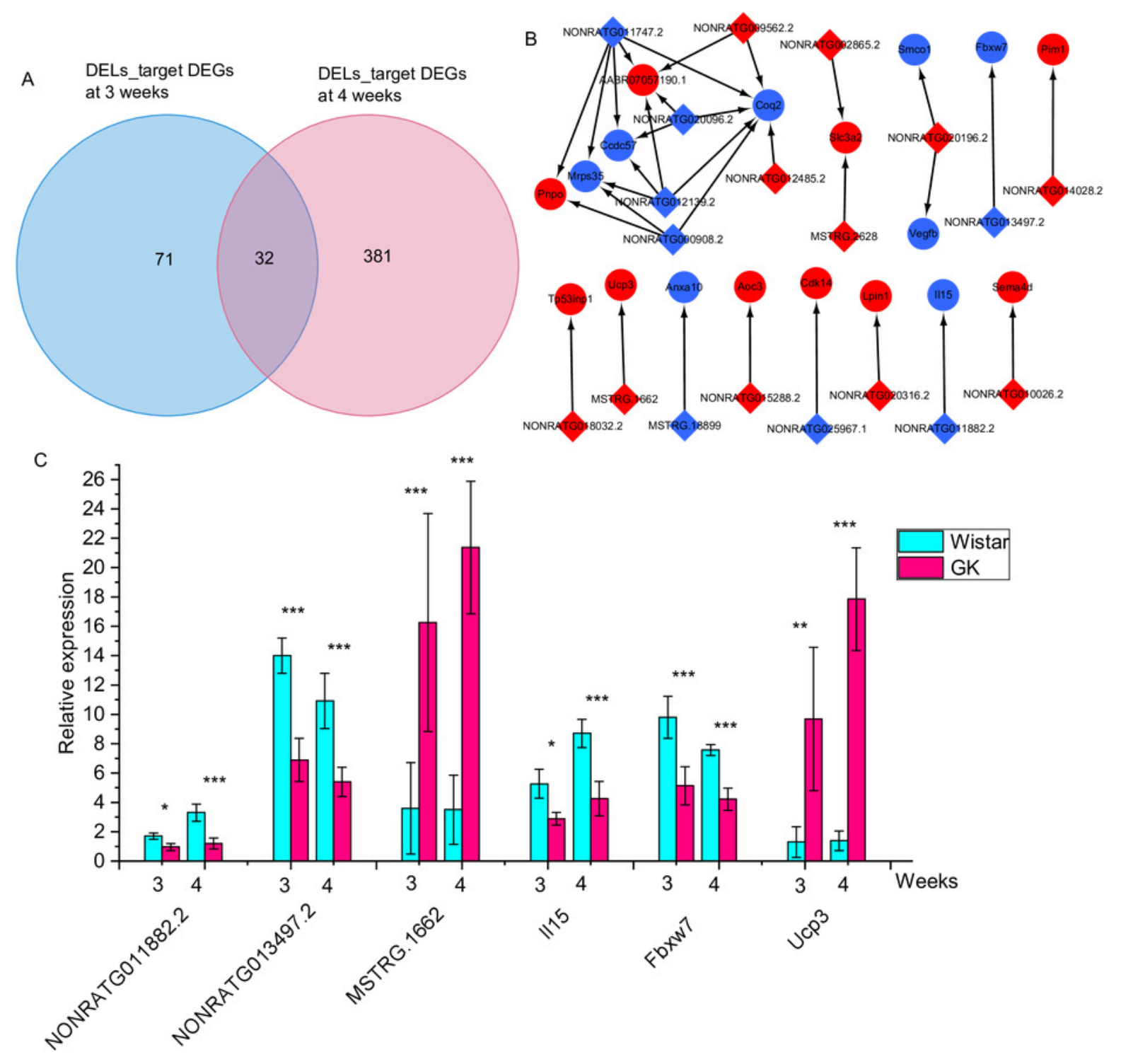




\section{Figure 6}

Figure 6 The target miRNAs of DEGs and DELs in key IncRNA-mRNA pairs.

The light orange represents IncRNA. The light blue represents miRNA. The light green represents mRNA.
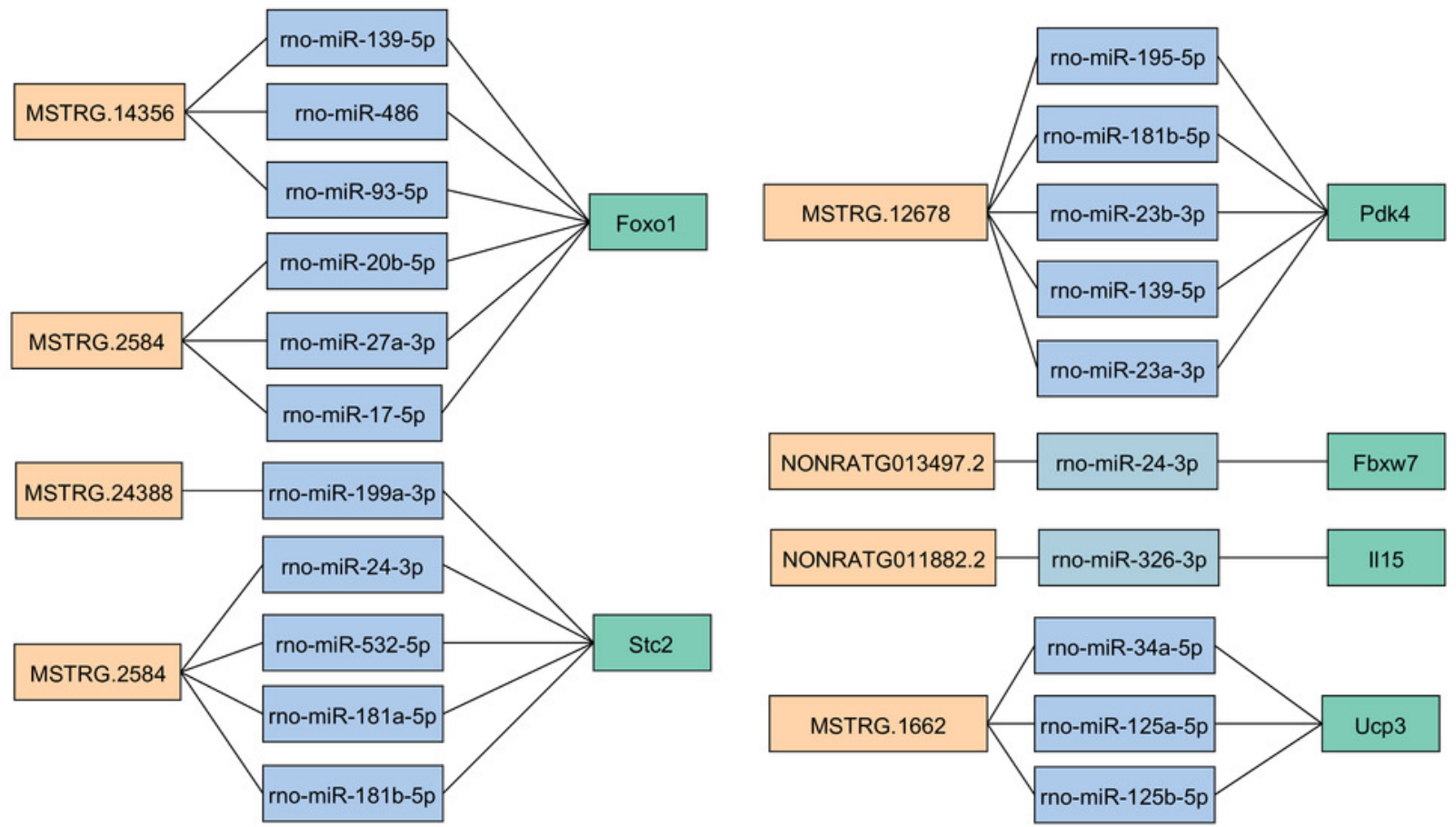


\section{Table $\mathbf{1}$ (on next page)}

Table 1 The characteristics of rats.

Values are means $\pm \mathrm{SD},{ }^{* * *} P<0.001$ vs age-matched Wistar group, $\mathrm{n}=10$ 
1 Table 1 The characteristics of rats

\begin{tabular}{ccccc}
\hline Rats & Age, weeks & Plasma glucose, $\mathbf{~ m m o l} / \mathbf{L}$ & Plasma insulin, $\mathbf{p m o l} / \mathbf{L}$ & Weight, $\mathbf{g}$ \\
\hline Wistar & 3 & $5.05 \pm 0.39$ & $157.66 \pm 64.52$ & $88.92 \pm 7.08$ \\
& 4 & $6.65 \pm 0.49$ & $168.97 \pm 36.37$ & $139.42 \pm 9.62$ \\
GK & 3 & $7.82 \pm 0.43 * * *$ & $197.83 \pm 57.69$ & $85.03 \pm 14.36$ \\
& 4 & $11.13 \pm 0.29 * * *$ & $147.16 \pm 59.92$ & $131.94 \pm 16.56$ \\
\hline
\end{tabular}


Table 2 (on next page)

Table 2 Top 10 nodes ranked by the degree in co-expressed IncRNA-mRNA network. 
1 Table 2 Top 10 nodes ranked by the degree in co-expressed lncRNA-mRNA network.

\begin{tabular}{lll}
\hline ID (Name) & Type & Degree \\
\hline ENSRNOG00000017286 (Ephx2) & mRNA & 34 \\
ENSRNOG00000005177 (Tp53i3) & mRNA & 33 \\
ENSRNOG00000026493 (Cdnf) & mRNA & 32 \\
MSTRG.2584 & IncRNA & 31 \\
ENSRNOG00000024924 (Cep19) & mRNA & 31 \\
ENSRNOG00000000473 (Pfdn6) & mRNA & 30 \\
MSTRG.8694 & lncRNA & 28 \\
MSTRG.14356 & lncRNA & 26 \\
ENSRNOG00000010802 (Ube3d) & mRNA & 25 \\
ENSRNOG00000016937 (Mtfrll) & mRNA & 25 \\
\hline
\end{tabular}

2 